\title{
DANOS ANATÔMICOS AO ESFÍNCTER ESOFÁGICO INFERIOR APÓS GASTRECTOMIA SUBTOTAL
}

\author{
ANATOMIC DAMAGE OF THE LOWER ESOPHAGEAL SPHINCTER AFTER SUBTOTAL GASTRECTOMY

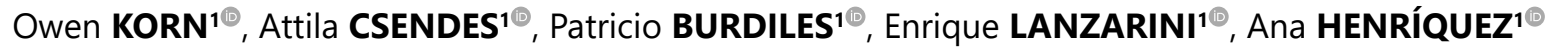

\begin{abstract}
RESUMO-RACIONAL: Disfunção do esfíncter esofágico inferior (EEI), doença do refluxo gastroesofágico e esofagite erosiva em pacientes submetidos à gastrectomia subtotal são ocorrências comumente reconhecidas, mas até agora as causas permanecem obscuras. OBJETIVO: A hipótese deste estudo é que a gastrectomia subtotal provoque alterações na pressão de repouso do $\mathrm{EEI}$ e na sua competência, devido ao dano anatômico desta, visto que as fibras oblíquas "Sling", um dos componentes musculares do EEI, são seccionadas durante este procedimento cirúrgico. MÉTODOS: Sete cães adultos sem raça definida $(18-30 \mathrm{~kg})$ foram anestesiados e submetidos à transecção do estômago proximal. Em seguida, o remanescente gástrico proximal foi fechado por sutura. No intraoperatório, manometria lenta foi realizada em cada cão, em condições basais (com estômago intacto) e no remanescente gástrico proximal fechado. A média dessas medidas é apresentada, com cada cão servindo como seu próprio controle. RESULTADOS: A pressão média do EEI medida no remanescente gástrico proximal, em comparação com a pressão do EEl no estômago intacto, foi diminuída em cinco cães, aumentada em um cão e sem alterações no outro cão. CONCLUSÃO: A secção transversa superior do estômago e o fechamento do remanescente do estômago por sutura provocam alterações na pressão do EEl. Sugerimos que essas mudanças na pressão do EEl são secundárias à secção das fibras oblíquas "Sling" do esfíncter, um de seus componentes musculares. A sutura e o fechamento do remanescente gástrico proximal, reancora essas fibras com mais, menos ou a mesma tensão, modificando ou não a pressão do EEl.
\end{abstract}

DESCRITORES: Refluxo gastroesofágico. Esfíncter esofágico inferior. Gastrectomia.

ABSTRACT - BACKGROUND: Dysfunction of the lower esophageal sphincter (LES), gastroesophageal reflux disease, and erosive esophagitis in patients undergoing subtotal gastrectomy are commonly recognized occurrences, but until now the causes remain unclear. AIM: The hypothesis of this study is that subtotal gastrectomy provokes changes on the LES resting pressure and its competence, due to the anatomical damage of it, given that the oblique "Sling" fibers, one of the muscular components of the LES, are transected during this surgical procedure. METHODS: Seven adult mongrel dogs $(18-30 \mathrm{~kg}$ ) were anesthetized and admitted for transection of the proximal stomach. Later, the proximal gastric remnant was closed by a suture. Intraoperatively, slow pull-through LES manometries were performed on each dog, under basal conditions (with the intact stomach), and in the closed proximal gastric remnant. The mean of these measurements is presented, with each dog serving as its control. RESULTS: The mean LES pressure (LESP) measured in the proximal gastric remnant, compared with the LESP in the intact stomach, was decreased in five dogs, increased in one dog, and remained unchanged in other dogs. CONCLUSION: The upper transverse transection of the stomach and closing the stomach remnant by suture provoke changes in the LESP. We suggested that these changes in the LESP are secondary to transecting the oblique "Sling" fibers of the LES, one of its muscular components. The suture and closing of the proximal gastric remnant reanchor these fibers with more, less, or the same tension, whether or not modifying the LESP.

HEADINGS: Gastroesophageal reflux. Esophageal sphincter, lower. Gastrectomy.

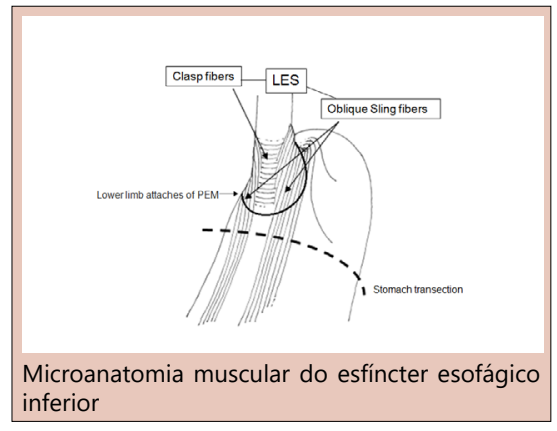

\begin{tabular}{|l|}
\hline Mensagem central \\
\hline Literatura mais antiga e recente é bem conhecido \\
que pacientes submetidos à gastrectomia distal \\
ou subtotal podem desenvolver esofagite ou \\
doença do refluxo gastroesofágico no pós- \\
operatório. Alguns autores sugeriram que isso se \\
deve à disfunção do esfíncter esofágico inferior
\end{tabular}

\begin{tabular}{|l|}
\hline Perspectiva \\
\hline Neste estudo experimental, nossa hipótese é que \\
a gastrectomia subtotal pode modificar a pressão \\
do esfíncter esofágico inferior em consequência \\
do dano anatômico ao próprio esfíncter, visto que \\
a secção transversa do estômago no terço médio \\
superior corta suas fibras musculares oblíquas \\
(fibras de estilingue - sling fibers ) que são um dos \\
componentes musculares do esfíncter.
\end{tabular}

\section{Perspectiva}

Neste estudo experimental, a gastrectomia subtotal pode modificar a pressão esfincter esofágico inferior em consequencia a secção transversa do estômago no terço médio superior corta suas fibras musculares oblíquas componentes musculares do esfíncter.

\section{Correspondência:}

Owen Korn.

E-mail: owenkorn@gmail.com; okorn@hcuch.c
Conflito de interesse: Nenhum

Financiamento: Nenhum

Recebido: $23 / 04 / 2021$

Aceito: 02/08/2021 


\section{INTRODUÇÃO}

Na literatura cirúrgica mais antiga e recenteé bem conhecido que uma porcentagem de pacientes submetidos à gastrectomia distal ou subtotal pode desenvolver esofagite ou doença do refluxo gastroesofágico (DRGE) no pós-operatório 1,4,10,11,15,19,25. Alguns autores sugeriram que isso se deve à disfunção do esfíncter esofágico inferior (EEI) e, embora existam várias teorias, até o momento, a causa dessa disfunção não está clara ${ }^{10,11,14,24,26}$.

Neste estudo experimental, nossa hipótese é que a gastrectomia subtotal pode modificar a pressão do EEI em consequência do dano anatômico ao próprio EEl, visto que a secção transversa do estômago no terço médio superior corta suas fibras musculares oblíquas (fibras de estilingue -Sling fibers) que são um dos componentes musculares do $\mathrm{EEI}^{17}$.

\section{MÉTODOS}

Este estudo experimental foi realizado em sete cães adultos sem raça definida (duas fêmeas e cinco machos) pesando $20-30 \mathrm{~kg}$. Este modelo animal tem sido utilizado historicamente em estudos manométricos experimentais do EEl, pois a anatomia regional dele em cães é bastante comparável à anatomia humana, e, portanto, constitui um modelo validado $0^{6,8,18,20}$. Protocolos institucionais de cuidado com animais para pesquisa foram rigorosamente seguidos, e cuidado especial foi tomado para prevenir qualquer dor ou estresse nos animais. Após jejum de $8 \mathrm{~h}$, os cães foram anestesiados com tiopental sódico intravenoso, intubados e oxigenados porventilação controlada. Nenhum relaxante muscular foi administrado e bolus de Fentanil $5 \mu \mathrm{g} / \mathrm{kg}$ foi aplicado para controle da dor.

Cuidados especiais foram tomados para manter oxigenação satisfatória, pressão arterial normal e sangramento mínimo para evitar qualquer efeito desses fatores sobre a pressão do $\mathrm{EE}^{3,16}$. Após laparotomia mediana, a manometria estacionária do EEI foi realizada em todos os cães sob duas condições. A primeira com o estômago intacto, que foi considerada a medida basal. Em seguida, o estômago foi seccionado transversalmente no terço superior, iniciando na curva menor $3 \mathrm{~cm}$ distal à inserção gástrica da membrana frenoesofágica (Figura 1). Após o fechamento do remanescente gástrico com sutura contínua em uma camada, foi realizada a segunda manometria.

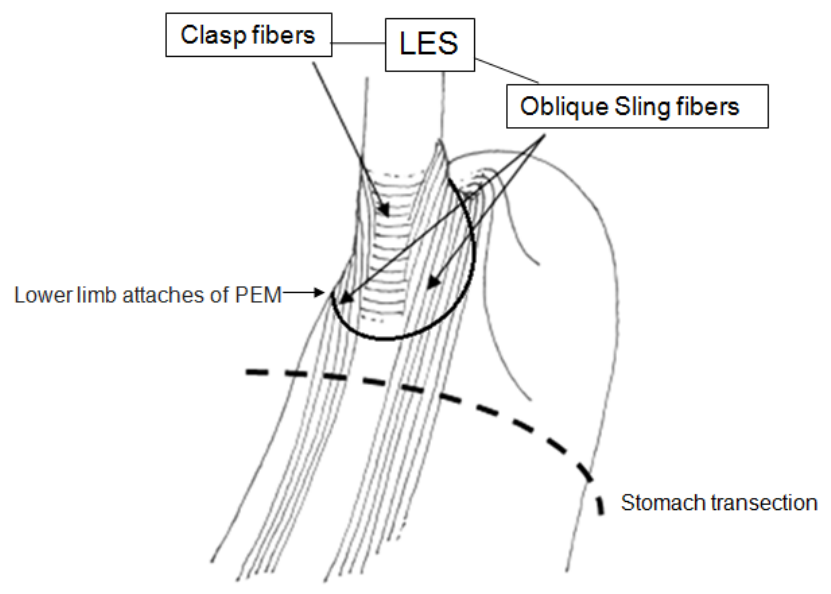

Figura 1 - Representação esquemática do EEl, as fibras curtas semicirculares "Clasp" na curva menor na cárdia e as fibras oblíquas longas "Sling", estendendo-se da cárdia no lado da grande curvatura, correndo em paralelo à curva menor para dentro o antro gástrico. A linha pontilhada mostra o nível de transecção do estômago no modelo experimental, distal à fixação inferior da membrana pré-esofágica (PEM)
Esses estudos manométricos foram realizados com um dispositivo com quatro cateteres de polivinil unidos com os orifícios distais laterais dispostos radialmente no mesmo nível e quatro aberturas proximais espaçadas a cada $5 \mathrm{~cm}$ (Synectics Medical, Estocolmo, Suécia). Eles foram perfundidos continuamente com $\mathrm{NaCl}$ a 0,9\% de uma bomba hidropneumocapilar a uma taxa de 0,5 ml/min (Arndorfer Medical Specialties, Milwaukee, WI) e conectados a um sistema de manometria computadorizada com quatro canais (Synectics Medical, Suécia). Antes de cada teste, o sistema era calibrado e a oclusão de qualquer orifício lateral produzia aumento de $400 \mathrm{mmHg}$ em $1 \mathrm{~s}$. Em cada condição, três retiradas lentas de pull-through foram realizadas. Devido à assimetria das pressões esfincterianas, cada retirada proporcionou pressões diferentes em cada canal e, portanto, a média desses quatro valores foi considerada como a pressão final do EEl para cada retirada. A pressão gástrica fúndica foi considerada como referência zero. Na análise dos resultados, cada cão era seu próprio controle.

\section{RESULTADOS}

Oprimeiro registro manométrico foi a pressão intraoperatória do EEI em repouso para cada cão e foi tomada como linha de base, ou seja, com o abdome aberto, mas com o estômago intacto. A pressão observada do EEl variou entre 9,6 e 15,9 $\mathrm{mmHg}$ em seis animais. Um cão apresentou pressão basal que era o dobro desses valores $(30,5 \mathrm{mmHg}$, Tabela 1$)$.

Tabela 1 - Medição da pressão EEl em cada cão sob condições experimentais (Cada cão era seu próprio controle)

\begin{tabular}{|lcc|}
\hline Cão & $\begin{array}{r}\text { Estômago intacto } \\
\text { EEI }(\mathbf{m m H g}) \mathbf{S D}\end{array}$ & $\begin{array}{r}\text { Remanescente gástrico fechado } \\
\text { EEI }(\mathbf{m m H g} \mathbf{m} \mathbf{H} \mathbf{S D}\end{array}$ \\
\hline 1 & $15,5 \pm 2,1$ & $11,7 \pm 4,0$ \\
\hline 2 & $10,2 \pm 0,7$ & $7,5 \pm 1,0$ \\
\hline 3 & $13,3 \pm 0,4$ & $5,0 \pm 2,0$ \\
\hline 4 & $15,9 \pm 1,8$ & $11,6 \pm 2,8$ \\
\hline 5 & $14,5 \pm 5,0$ & $4,3 \pm 0,6$ \\
\hline 6 & $9,6 \pm 1,4$ & $9,3 \pm 3,0$ \\
\hline 7 & $30,5 \pm 1,0$ & $32,5 \pm 3,5$ \\
\hline
\end{tabular}

Depois de fechar o remanescente gástrico proximal com uma sutura contínua, uma nova manometria era realizada, e as alterações na pressão do EEl foram registradas em cada cão e comparadas com a pressão basal desse mesmo cão. $A$ pressão média final do EEl observada foi diminuída em cinco cães (Figura 2), permaneceu inalterada em um (cão 6) e em um cão a pressão foi maior do que sua linha de base (cão 7).

\section{DISCUSSÃO}

Este estudo experimental foi desenhado para avaliar eventualmente mudanças na pressão do EEl em cães após a secção do estômago proximal, tentando reproduzir o método em outros relatos na literatura que estudaram a pressão dele em pacientes antes e depois de serem submetidos à gastrectomia distal ${ }^{10,11,14,19,24,26}$.

Historicamente, uma questão muito discutida que persiste até hoje é entender como funciona o EEI e compreender e aceitar sua anatomia única ${ }^{7}$. Há muito tempo a existência de um EEI anatômico tem sido questionada, pois a anatomia regional não parece mostrar estrutura muscular que satisfaça a definição arbitrária de um esfíncter, como um "anel" muscular². 


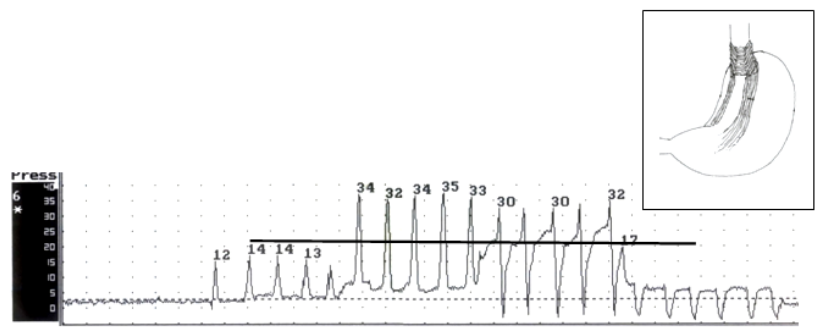

A) Intact stomach

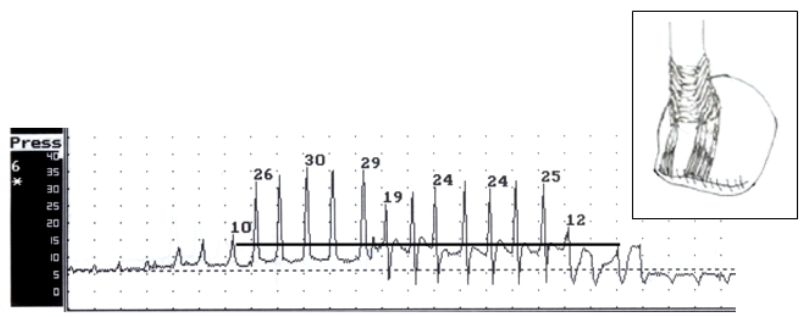

B) Sectioned and sutured stomach (closed gastric remnant)

Figura 2 - Traçados de pressão do EEl obtidos em condições experimentais no intraoperatório (cão 4): A) pressão do EEl na condição basal com o estômago intacto; B) diminuição da pressão após a transecção do estômago e fechamento do remanescente gástrico.

Assim, sem um correlato anatômico, mas com a nítida demonstração manométrica de um esfíncter gastroesofágico por Fike et al. ${ }^{5}$ em 1956, iniciou-se discussão sobre a existência de um "esfíncter fisiológico", o que dificultou nossa compreensão de seu comportamento. O trabalho de Liebermann-Meffert no final dos anos 1970 confirmou o que os antigos anatomistas haviam mostrado, ou seja, que na cárdia não existe uma estrutura muscular como um anel, mas há duas bandas musculares dispostas perpendicularmente nela: o músculo EE $\left.\right|^{17}$. Apesar dessa descrição anatômica detalhada, ele permaneceu conceitualmente na mente de muitos pesquisadores como um anel muscular. Essa interpretação errônea da anatomia do LES tem impedido o entendimento e a explicação sobre as peculiaridades do comportamento desse esfíncter com sua estrutura anatômica particular ${ }^{20}$.

A causa do desenvolvimento da DRGE e/ou esofagite em alguns pacientes submetidos à gastrectomia parcial distal permanece obscura ${ }^{1,4,10,11,15,19,23}$. Alguns autores sugeriram que a ressecção do estômago distal provoca alterações nos níveis de certos níveis de hormônios gastrointestinais que modificariam a pressão do $\mathrm{EEI}^{26}$. Outros argumentam que a lesão da membrana frenoesofágica, bem como alterações do ângulo esofagogástrico (His) relacionadas à reconstrução da continuidade gastrointestinal, podem alterar a função esfincteriana $a^{4,14,19,22,23}$, alguns atribuindo a origem dessa disfunção do EEl ao tipo de reconstrução, seja do tipo Billroth I ou II ${ }^{4,11}$

No entanto, as explicações oferecidas acima não são convincentes. Está estabelecido que nem sempre ocorre refluxo gastroesofágico ou disfunção esfincteriana e que essa disfunção nem sempre é a mesma. Se a ressecção do estômago distal causar alterações hormonais, seria de se esperar que ocorresse com mais frequência do que o observado. Em contraste, após gastrectomia distal, geralmente não ocorrem lesões na membrana frenoesofágica ou alterações no ângulo de His. Portanto, essas teorias não fornecem explicação razoável para suas descobertas.

O estudo de Lida et al. ${ }^{11}$ é particularmente intrigante e desafia as teorias acima. Esses autores mediram a pressão do EEl em repouso em 42 pacientes, antes e depois de uma gastrectomia distal, descobrindo que em 21 pacientes $(50 \%)$ a pressão do EEl permaneceu inalterada, enquanto que em 17 pacientes (40\%) a pressão diminuiu, e em quatro (10 \%) houve aumento consistente na pressão. Os autores descrevem esses achados, sem dar explicação satisfatória para suas causas.

A hipótese proposta e testada neste estudo experimental é baseada na estrutura anatômica do EEI descrita por LiebermannMeffert et al. ${ }^{17}$ e verificada por estudos de nosso grupo ${ }^{12,13}$. O EEl é formado por duas bandas de músculos dispostos quase perpendicularmente que atuam de maneira complementar para fechar a cárdia: as fibras musculares semicirculares ou "pinça" na curva menor e as fibras musculares oblíquas ou "fibras de estilingue/sling" na lateral da grande curvatura. As fibras do sling se estendem do esôfago distal e fundo gástrico proximal e correm paralelas à curva menor no antro gástrico (Figura 1).

Por este motivo, a secção transversal do terço superior do estômago ou mesmo em seu terço médio, por necessidade corta as fibras musculares oblíquas "sling" e, portanto, um dos componentes do esfíncter fica gravemente danificado $0^{9,12,13,17,18,21}$. Quando a continuidade gastrointestinal é restaurada, por meio de uma anastomose gastroduodenal (Billroth I) ou gastrojejunal (Billroth II ou Y-de-Roux), ou o remanescente gástrico é fechado (neste estudo experimental) pela sutura, as fibras oblíquas "sling" são re-ancoradas. Porém, esse reparo nem sempre restaura a simetria ou a tensão dessas fibras que existiam antes da transecção e do fechamento do remanescente, o que pode modificar a pressão de repouso do $\mathrm{EE}^{24}$.

Com base em nossos resultados e nos referidos conceitos anatômicos, sustentamos que é possível explicar os diferentes efeitos sobre o EEl, observados após a gastrectomia distal ${ }^{10,11,19}$. As fibras oblíquas em pacientes podem ser reancoradas com três resultados possíveis: 1) a mesma simetria e tensão de antes da transecção gástrica, restaurando assim a pressão e função normais do $\mathrm{EEl}$; 2) com menos tensão criando um esfíncter hipotenso e eventualmente incompetente; ou 3) as fibras podem ser suturadas com maior tensão e aperto, resultando em um esfíncter hipertensivo.

Algumas limitações desta investigação são o baixo número de cães (apenas sete), condições experimentais agudas, espécimes sob anestesia geral e a falta de um grupo controle.

\section{CONCLUSÃO}

Os achados deste estudo experimental em cães parecem reproduzir as observações clínicas no homem, e dar explicação razoável, para as diferentes mudanças na pressão de repouso do EEl observadas em pacientes após gastrectomia distal. Acreditamos que essesachadosfornecem resposta anatomicamente efisiologicamente consistente a um antigo mistério da cirurgia gástrica.

\section{AGRADECIMENTOS}

Gostaríamos de agradecer à Sra. Alejandra Vallebona e ao Dr. Michael G. Sarr, MD, pela revisão deste artigo.

\section{REFERÊNCIAS}

1. Braghetto I, Velasco N, Csendes A, et al. Gastroesophageal reflux in gastric and duodenal ulcer patients before and after definitive surgical treatment: A prospective study. In Esophageal Disorders: Pathophysiology and Therapy. TR DeMesster, DB Skinner (Eds) Raven Press, New York, 1985, 155 - 163.

2. Didio LJA, Anderson MC (Eds). The sphincters of the digestive system. Anatomical, functional and surgical considerations. Baltimore, Williams \& Wilkins, 1968. 
3. Earlam RJ, Schlegel JF, Ellis FH Jr. Effect of ischemia of lower esophagus and esophagogastric junction on canine esophageal motor function.J Thorac Cardiovasc Surg 1967;54(6):822-831.

4. Endo A, Okamura S, Kono N, Katsumi M. Esophageal reflux after gastrectomy. A hazard after Billroth I subtotal gastrectomy. Int Surg 1978;63(4):52-58.

5. Code CF, Fyke FE Jr, Schlegel JF. The gastroesophageal sphincter in healthy human being. Gastroenterologia (Basel). 1956;86(3):135150. doi: $10.1159 / 000200544$.

6. Franzi SJ, Martin CJ, Cox MR, Dent J. Response of canine lower esophageal sphincter to gastric distension. Am J Physiol 1990;259(3 Pt 1):G380-385. doi: 10.1152/ajpgi.1990.259.3.G380.

7. Friedland GW: Progress in radiology: Historical review of the changing concepts of the lower esophageal anatomy: 430 B.C. 1977. AJR Am J Roentgenol 1978;131(3):373-388.

8. Friedland GW, Kohatsu S, Lewin K. Comparative anatomy of feline and canine gastric sling fibers. Analogy to human anatomy. Am J Dig Dis. 1971;16(6):493 - 507. doi: 10.1007/BF02235539.

9. Gahagan T. The function of the musculature of the esophagus and stomach in the esophagogastric sphincter mechanism. Surg Gynecol Obst. 1962; 114:293 - 303.

10. Haga N; Mochiki E, Nakabayashi T, Suzuki T, Asao T, Kuwano $\mathrm{H}$. Esophageal manometric changes and gastroesophageal reflux symptoms after distal gastrectomy for gastric cancer. Hepatogastroenterology 2005; 52(61):310 -313. PMID: 15783057.

11. lida F, Ishizaka K, Sugenoya A. Loweresophageal sphincter pressure after subtotal gastrectomy and postoperative reflux esophagitis. Hepato-Gastroenterology 1994;41(6):581 - 584. PMID: 7721250.

12. KornO,Stein HJ, RichterTH, Liebermann-MeffertD.Gastroesophageal sphincter:a model. Dis Esophagus 1997;10(2):105-109. doi:10.1093/ dote/10.2.105.

13. Korn O, Csendes A, Burdiles P, Braghetto I, Stein HJ. Anatomic dilatation of the cardia and competence of the lower esophageal sphincter: a clinical and experimental study. J Gastrointest Surg. 2000;4(4):398 - 406. doi: 10.1016/s1091-255x(00)80019-0.

14. Kujiwara Y, Nakagawa K, Kusunoki M, Tanaka T, Yamamura T, UtsunomiyaJ.Gastroesophageal refluxafterdistal gastrectomy:possible significance of the angle of His. Am J Gastroenterol 1998;93(1):11 15. doi: 10.1111/j.1572-0241.1998.011_c.x. PMID: 9448165.

15. Lataste J, Gouthier F. Le refluxaesophagiens aprés gastrectomies distales. La PresseMed. 1967; 75: 1025 - 1028.
16. Licalzi LK, Biancani P, Behar J, Kerstein MD. Effect of hemorrhagic hypotension and hypoventilation on lower esophageal sphincter pressure.. Ann Surg 1980;192(1):53 - 57. doi: 10.1097/00000658198007000-00009.

17. Liebermann-Meffert $D$, Allgöwer $M$, Schmid P, Blum AL. Muscular equivalent of the lower esophageal sphincter. Gastroenterology 1979;76(1):31 - 38. PMID: 81791.

18. Liemm HH, Martin CJ. The contribution of the gastric sling to canine gastroesophageal competence. Gastroenterology 1993; 104: A543.

19. Marcovechio CA, MartinezJC, PiesciottoA, Yanagita ET, Denardin OVP, Herani Filho B. Manometric study of the lower esophageal sphincter and esophagus in subtotal gastrectomy patients. Dis Esophagus 2008; 21(2):118-124. doi: 10.1111/j.1442-2050.2007.00734.x.

20. Mariotto R, Herbella FAM, Andrade VLÂ, Schlottmann F, Patti MG. Validation of a new water-perfused high-resolution manometry system. Arq Bras Cir Dig. 2021;33(4):e1557. doi: 10.1590/0102$672020200004 \mathrm{e} 1557$.

21. Meiss JH, Grindlay JH, Ellis FH, Jr. The gastroesophageal sphincter mechanism. II. Further experimental studies in the dog. J Thoracic Surg 1958;36(2):156 - 165.

22. Santoro S, Aquino CGG, Mota FC, Artoni RF. Does evolutionary biology help the understanding of metabolic surgery? a focused review. Arq Bras Cir Dig. 2020;33(1):e1503. doi: 10.1590/0102$672020190001 \mathrm{e} 1503$.

23. Schneider J, Becker $H$, Lepsien $G$. The experimental effect of myectomy of the ventral obliquae sling fibres on the lower esophageal sphincter pressure. In Giuli R, McCallum R, Skinner D, (Eds.) Primary Motility Disorders of the Esophagus. John Libbey Eurotext, Paris, 1991, 461 - 466.

24. Takahashi T, Yoshida M, Kubota T, Otani Y, Saikawa Y, Ishikawa H, Suganuma K, Akatsu Y, Kumai K, Kitajima M. Morphologic analysis of gastroesophageal reflux diseases in patients after distal gastrectomy. World J. Surg 2005; 29(1):50 - 57. doi: 10.1007/ s00268-004-7415-3.

25. Windsor CW. Gastro-esophageal reflux after partial gastrectomy. BrMed J.1964;2(5419): 1233-1234. doi:10.1136/bmj.2.5419.1233.

26. Yamashita $Y$, Inoue H, Ohta K, Yoshida K, Miyahara E, Hirai T, Toge T, Adrian TE. Manometric and hormonal changes after distal partial gastrectomy. Aliment Pharmacol Ther 2000;14 (Suppl 1):166 - 169. doi: 10.1046/j.1365-2036.2000.014s1166.x. 